\title{
Original
}

\section{Association of serum YKL-40 levels with urinary albumin excretion rate in young Japanese patients with type 1 diabetes mellitus}

\author{
Fumie Sakamoto ${ }^{1)}$, Naoto Katakami ${ }^{1), 2)}$, Hideaki Kaneto ${ }^{1)}$, Tetsuyuki Yasuda ${ }^{1)}$, Mitsuyoshi Takahara1), \\ Kazuyuki Miyashita $^{1)}$, Akio Kuroda ${ }^{3)}$, Munehide Matsuhisa ${ }^{3)}$, Keisuke Kosugi ${ }^{4)}$ and Iichiro Shimomura ${ }^{1)}$ \\ 1) Department of Metabolic Medicine, Osaka University Graduate School of Medicine, Suita 565-0871, Japan \\ 2) Department of Metabolism and Atherosclerosis, Osaka University Graduate School of Medicine, Suita 565-0871, Japan \\ 3) Diabetes Clinical Research and Development Center, Tokushima University, Tokushima 770-8503, Japan \\ 4) Department of Internal Medicine, Osaka Police Hospital, Osaka 543-0035, Japan
}

\begin{abstract}
YKL-40 is a marker of inflammation and endothelial dysfunction, both of which play important roles in the progression of diabetic complications. However, little information has been obtained about serum YKL-40 levels in type 1 diabetic patients. We evaluated YKL-40 levels and its association with diabetic micro- and macroandgiopathy in 131 young Japanese type 1 diabetic patients without advanced diabetic complications (aged 24.7 \pm 5.9 years) and 97 age- and gendermatched healthy controls. YKL-40 levels were significantly elevated in type 1 diabetic patients than in healthy controls (median (range) 46.4 (20.3-136.7) and 52.3 (21.4-274.1) ng/mL, respectively, $p=0.003$ ). There was a significant positive association between YKL-40 levels and urinary albumin creatinine ratio (UACR) $(r=0.226, p=0.013)$. Furthermore, a multivariate regression analysis demonstrated that YKL-40 levels were a determinant of UACR independently of conventional risk factors. In addition, YKL-40 levels were significantly higher in participants with diabetic retinopathy compared to those without it (median (range) 55.5 (23.3-274.1) and $50.3(21.4-237.4) \mathrm{ng} / \mathrm{mL}$, respectively, $p=0.039$ ). Serum YKL-40 levels were elevated in type 1 diabetic patients and associated with increasing level of albuminuria. YKL40 could be a predictor to assess the risk of diabetic microangiopathy in the early stage in type 1 diabetic patients.
\end{abstract}

Key words: YKL-40, Type 1 diabetes, Albuminuria, Diabetic retinopathy

DIABETIC micro- and macrovascular complications, which are accelerated in patients with prolonged type 1 diabetes mellitus even in their youth, lead to the impairment of quality of life and are major causes of mortality. Therefore, it is important to identify some predictors of diabetic complications in their early stage. Despite exhausting efforts, we have not yet identified any markers to predict the prognosis in early stage of diabetes.

YKL-40, also known as chitinase-3-like-1 protein $(\mathrm{CHI} 3 \mathrm{~L} 1)$, is reported to be a marker involved in inflammation and endothelial dysfunction, both of which play important roles in the progression of dia-

Submitted Jul. 2, 2012; Accepted Aug. 30, 2012 as EJ12-0238 Released online in J-STAGE as advance publication Sep.15, 2012

Correspondence to: Hideaki Kaneto, M.D., Ph.D., Department of Metabolic Medicine, Osaka University Graduate School of Medicine, 2-2, Yamadaoka, Suita, Osaka 565-0871, Japan.

E-mail: kaneto@endmet.med.osaka-u.ac.jp betic complications. YKL-40 is a $40 \mathrm{kDa}$ heparin- and chitin-binding glycoprotein without chitinase activity, a member of the 'mammalian chitinase-like proteins'.

YKL-40 is secreted by a variety of cells such as activated macrophages and neutrophils in different tissues with inflammation, vascular smooth muscle cells, cancer cells and arthritic chondrocytes. Although the exact function of YKL-40 remains partly unknown, it is indicated to have an important role in inflammation, extracellular remodeling, fibrosis and angiogenesis [1]. Indeed, previous clinical studies have shown that YKL-40 levels were associated with various diseases and its activity or prognosis such as rheumatoid arthritis [2], cancer [1, 3], and cardiovascular disease [4].

Several studies have shown that YKL-40 levels were elevated in type 2 diabetic patients $[5,6]$. Furthermore, we previously demonstrated that there was a significant positive association between YKL-40 levels and albuminuria in these populations [7]. However, little infor- 
mation has been obtained about YKL-40 levels in type 1 diabetic patients [8].

The objective of this study was to evaluate serum YKL-40 levels and its association with diabetic microand macroangiopathy in young Japanese type 1 diabetic patients without advanced diabetic complications.

\section{Materials and Methods}

\section{Study population}

Young Japanese type 1 diabetic patients who had annual periodic examinations in Osaka University Medical Hospital or Osaka Police Hospital were recruited in the present study. The diagnosis of type 1 diabetes mellitus was made by diabetologists when hyperglycemia and/or ketosis were observed, and since then all patients have received insulin therapy. The participants with neoplasm, liver dysfunction, autoimmune disease, acute infections, cardiovascular disease or use of antihypertensive and lipid lowering drugs were excluded. After all, 131 type 1 diabetic patients and 97 age- and gendermatched healthy volunteers were enrolled. The study was approved by the local ethics committee and was conducted in accordance with the Helsinki Declaration. All participants provided written informed consent.

\section{Clinical and biochemical assessment}

The clinical examination and collection of blood and urine samples were performed. The clinical examination included a medical history interview, physical examination (measurement of height, weight and resting blood pressure), electrocardiography, coefficient of variation of R-R intervals (CVR-R), assessment of carotid intima-media thickness by ultrasonographic imaging as previously reported [9], and fundus photography. Smoking status was classified as having a current smoking habit or not.

Venous blood was taken after a $12 \mathrm{~h}$ fasting state and the following laboratory analyses were performed by SRL Inc. (Tokyo, Japan). HbA1c levels were measured by latex agglutination turbidimetry using a set of calibrators assigned by the Japan Diabetes Society (JDS) (normal range 4.3-5.8\%). The value for HbAlc was estimated as a National Glycohemoglobin Standardization Program equivalent value calculated with the formula: HbAlc $(\%)=$ HbAlc $(J D S)(\%)+0.4 \%$ [10]. Plasma glucose, serum total cholesterol, high density lipoprotein (HDL) cholesterol, triglyceride, creatinine and high sensitive C-reactive protein (hs-CRP) levels were mea- sured using standard laboratory protocols. Glomerular filtration rate (GFR) was estimated using the abbreviated Modification of Diet in Renal Disease (MDRD) equation by 0.881 for Japanese [11]. After 24h urine collections, albumin concentration was measured by tubidimetric immunoassay, and then calculated urinary albumin to creatinine ratio (UACR). Presence of retinopathy was diagnosed by a diabetologist based on the findings of single-field fundus photography and classified as non diabetic retinopathy (NDR) and simple or more advanced diabetic retinopathy $(\geq \mathrm{SDR})$.

\section{Measurement of serum YKL-40 levels}

Serum YKL-40 levels were analyzed with a commercial enzyme immunoassay (QUIDEL Corp., San Diego, CA, USA) on material frozen at the time of inclusion. Serum was stored at $-30{ }^{\circ} \mathrm{C}$ until analysis. The measuring range of the assay was $20-300 \mathrm{ng} / \mathrm{mL}$, and the intra-assay and inter-assay coefficients of variation were 5.8 and $6.0 \%$, respectively.

\section{Statistical analysis}

Normally distributed variables are given as mean \pm $\mathrm{SD}$, whereas variables with a non-Gaussian distribution were logarithmically transformed before analysis and are given as median (range). Comparisons between the groups were performed by an unpaired Student's $t$ test or Welch test. Differences in proportions were tested using the $\chi^{2}$-test or Fisher's exact test. Correlations between variables were tested by Pearson's univariate test. Multivariate regression analysis was performed to assess variables significantly associated with an objective variable. In case of multivariate regression analysis for categorical data, we performed logistic regression analysis to derive the odds ratio (OR) and 95\% confidence intervals $(\mathrm{CI})$.

A two-sided $p$ values $<0.05$ was considered statistically significant. All analyses were performed with the use of SAS software, version 9.2 (Cary, NC, USA).

\section{Results}

\section{Serum YKL-40 levels in type 1 diabetic patients and healthy controls}

Clinical characteristics and measurements of healthy control subjectss and type 1 diabetic patients are presented in Table 1. BMI, plasma glucose, HbA1c, serum triglyceride, creatinine levels and estimated GFR were significantly higher in type 1 diabetic patients compared 
Table 1 Clinical characteristics of type 1 diabetic patients and healthy controls

\begin{tabular}{|c|c|c|c|}
\hline & $\begin{array}{l}\text { Healthy } \\
\text { controls } \\
(\mathrm{n}=97)\end{array}$ & $\begin{array}{c}\text { Type } 1 \\
\text { diabetes } \\
(\mathrm{n}=131)\end{array}$ & $p$ \\
\hline Age (years) & $25.5 \pm 2.7$ & $24.7 \pm 5.9$ & 0.198 \\
\hline Gender (male / female) & $36 / 61$ & $50 / 81$ & 0.891 \\
\hline Duration of diabetes (years) & - & $13.3 \pm 6.6$ & - \\
\hline Smoking n (\%) & $19(19.6)$ & $30(24.4)$ & 0.419 \\
\hline BMI $\left(\mathrm{kg} / \mathrm{m}^{2}\right)$ & $20.5 \pm 2.7$ & $22.5 \pm 2.6$ & $<0.001$ \\
\hline Systolic BP (mmHg) & $114.0 \pm 12.4$ & $116.7 \pm 14.0$ & 0.135 \\
\hline Diastolic BP (mmHg) & $70.1 \pm 7.9$ & $70.3 \pm 9.7$ & 0.829 \\
\hline Glucose (mg/dL) & $84.7 \pm 6.1$ & $190.8 \pm 102.8$ & $<0.001$ \\
\hline $\mathrm{HbA} 1 \mathrm{c}(\%)$ & $5.0 \pm 0.2$ & $8.3 \pm 1.6$ & $<0.001$ \\
\hline $\mathrm{TC}(\mathrm{mg} / \mathrm{dL})$ & $180.3 \pm 23.4$ & $188.6 \pm 31.6$ & 0.342 \\
\hline Triglyceride (mg/dL) & $63(25-139)$ & $80(26-635)$ & 0.007 \\
\hline HDL-C (mg/dL) & $66.6 \pm 13.0$ & $69.9 \pm 17.0$ & 0.121 \\
\hline Creatinine (mg/dL) & $0.70 \pm 0.14$ & $0.66 \pm 0.13$ & 0.034 \\
\hline eGFR $\left(\mathrm{ml} / \mathrm{min} / 1.73 \mathrm{~m}^{2}\right)$ & $96.6 \pm 12.6$ & $105.1 \pm 16.9$ & $<0.001$ \\
\hline Urinary C peptide ( $\mu \mathrm{g} /$ day) & - & $2.2(0-38)$ & - \\
\hline TDD (units/kg) & - & $0.87 \pm 0.24$ & - \\
\hline CVR-R (\%) & - & $4.99 \pm 2.02$ & - \\
\hline Retinopathy (NDR / $\geq$ SDR) & - & $87 / 44$ & - \\
\hline $\mathrm{UACR}(\mathrm{mg} / \mathrm{g} \cdot \mathrm{Cr})$ & - & $6.41(1.8-752.5)$ & - \\
\hline Mean IMT (mm) & - & $0.58 \pm 0.09$ & - \\
\hline Max IMT (mm) & - & $0.72 \pm 0.13$ & - \\
\hline hs-CRP (ng/mL) & $171(53-7380)$ & $321(51-13000)$ & 0.001 \\
\hline
\end{tabular}

$\overline{\text { Data are means } \pm \mathrm{SD} \text {, median (range), or number unless specified }}$ otherwise. Unpaired $t$ test and Fisher's exact test were performed. $p$ value $<0.05$ was considered statistically significant.

BMI, body mass index; BP, blood pressure; HbAlc, glycated hemoglobin A1c; TC, total cholesterol; HDL-C, high density lipoprotein cholesterol; eGFR, estimated glomerular filtration rate; TDD, insulin total daily dose; CVR-R, coefficient of variation of $\mathrm{R}-\mathrm{R}$ intervals; NDR, non diabetic retinopathy; $\geq \mathrm{SDR}$, simple or more advanced diabetic retinopathy; UACR, urinary albumin to creatinine ratio

to control subjects $(p<0.05)$. There was no significant difference between the two groups regarding the other clinical parameters such as smoking status, systolic and diastolic blood pressures, and total cholesterol levels.

YKL-40 levels were significantly elevated in type 1 diabetic patients than control subjects (median (range) 46.4 (20.3-136.7) and 52.3 (21.4-274.1) ng/mL, respectively, $p=0.003$ ) (Fig. 1).

Correlation between serum YKL-40 levels and various factors in type 1 diabetic patients

YKL-40 levels showed significantly positive correlations with diabetes duration $(r=0.225, p=0.010)$

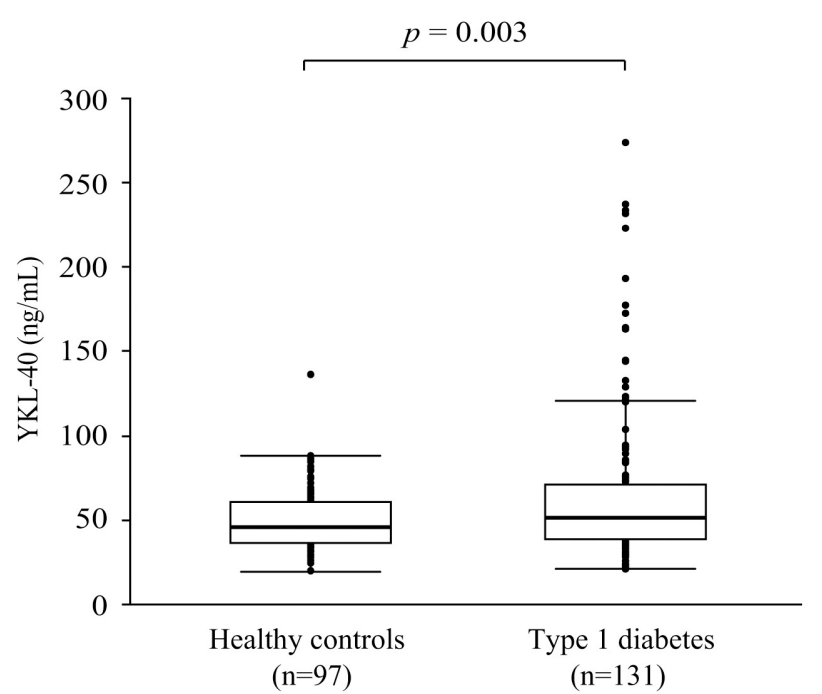

Fig. 1 Comparison of serum YKL-40 levels between type 1 diabetic patients and healthy controls: 46.4 (20.3-136.7) $\mathrm{ng} / \mathrm{ml}$ in the control groups, $52.3(21.4-274.1) \mathrm{ng} / \mathrm{mL}$ in the type 1 diabetic patients

Data are median levels (range).

and triglyceride levels $(r=0.337, p<0.001)$. Type 1 diabetic patients with a current smoking habit had significantly higher YKL-40 levels as compared to those without it (median (range) 50.8 (21.4-274.1) and 60.5 (23.3-237.4) $\mathrm{ng} / \mathrm{mL}$, respectively, $p=0.015)$. However, there was no statistically significant association between YKL-40 levels and the other variables including $\mathrm{HbA} 1 \mathrm{c}$ levels. A stepwise multivariate regression analysis including all variables that were significantly associated with YKL-40 levels in univariate analysis as independent variables (i.e. diabetes duration, smoking status and serum triglyceride levels) revealed that all these variables were independent determinants of YKL-40 levels (data not shown).

\section{Associations between serum YKL-40 levels and dia- betic complications}

Subsequently, we investigated the association between diabetic complications and YKL-40 levels. To examine a possible involvement of YKL-40 levels in the development of diabetic nephropathy, we evaluated the association between YKL-40 levels and UACR. There was a significant positive association between YKL-40 levels and UACR ( $r=0.226, p=0.013)$ (Fig. 2). UACR was also significantly correlated with diabetes duration $(r=0.189, p=0.038), \mathrm{HbA} 1 \mathrm{c}$ levels $(r=0.325, p<$ $0.001)$ and creatinine levels $(r=-0.181, p=0.048)$, and higher in female than in male (median (range) 7.5 


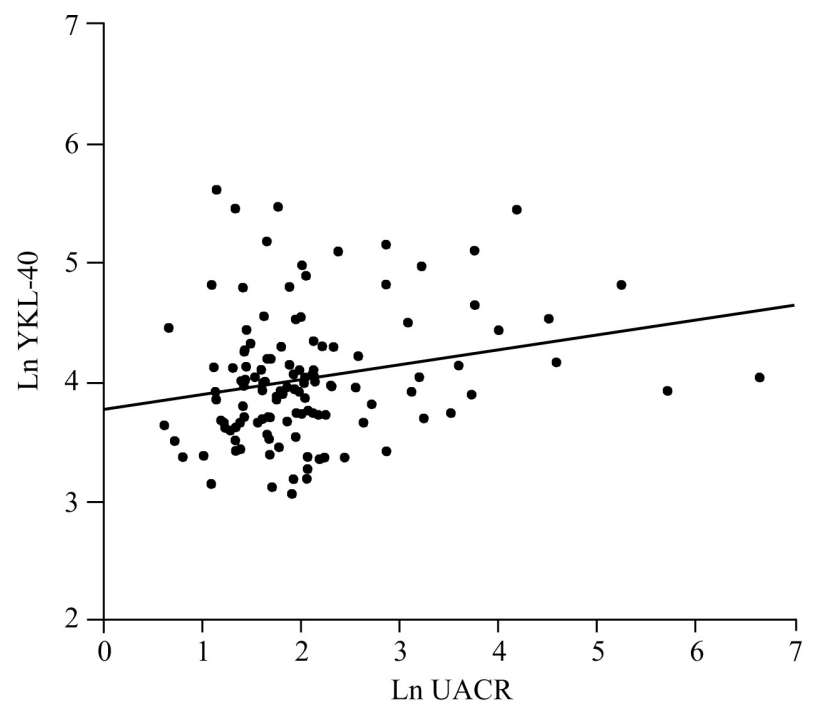

Fig. 2 Positive correlation between YKL-40 levels and UACR $(r=0.226, p=0.013)$

Data are logarithmically transformed. Statistical analysis for correlation was obtained by Pearson's univariate test. UACR, urinary albumin to creatinine ratio

Table 2 Correlation between urinary albumin to creatinine ratio and variables

\begin{tabular}{|c|c|c|c|c|}
\hline & \multicolumn{2}{|c|}{ Univariate } & \multicolumn{2}{|c|}{ Multivariate } \\
\hline & $r$ & $p$ & $\beta$ & $p$ \\
\hline Age (years) & 0.145 & 0.113 & & \\
\hline Gender $(0=$ female, $1=$ male $)$ & -0.285 & 0.002 & -0.270 & 0.002 \\
\hline Duration of diabetes (years) & 0.189 & 0.038 & 0.024 & 0.055 \\
\hline Smoking status $(0=$ no, $1=$ yes $)$ & 0.058 & 0.538 & & \\
\hline BMI $\left(\mathrm{kg} / \mathrm{m}^{2}\right)$ & -0.051 & 0.581 & & \\
\hline Systolic BP (mmHg) & -0.011 & 0.908 & & \\
\hline Diastolic BP (mmHg) & 0.110 & 0.232 & & \\
\hline Glucose (mg/dL) & -0.017 & 0.866 & & \\
\hline HbAlc $(\%)$ & 0.325 & $<0.001$ & 0.164 & 0.002 \\
\hline $\mathrm{TC}(\mathrm{mg} / \mathrm{dL})$ & 0.172 & 0.059 & & \\
\hline Triglyceride (mg/dL) & 0.047 & 0.610 & & \\
\hline HDL-C (mg/dL) & -0.017 & 0.854 & & \\
\hline Creatinine (mg/dL) & -0.181 & 0.048 & & \\
\hline $\mathrm{eGFR}\left(\mathrm{mL} / \mathrm{min} / 1.73 \mathrm{~m}^{2}\right)$ & -0.031 & 0.740 & & \\
\hline YKL-40 (ng/mL) & 0.226 & 0.013 & 0.369 & 0.018 \\
\hline
\end{tabular}

Stepwise multivariate regression analysis was performed to determine the predictors of UACR. The significant variables were used for multiple linear regression analysis in Pearson's univariate test. $\beta$ : partial regression coefficients

BMI, body mass index; BP, blood pressure; HbAlc, glycated hemoglobin A1c; TC, total cholesterol; HDL-C, high density lipoprotein cholesterol; eGFR, estimated glomerular filtration rate
$(1.8-752.5)$ and $4.8(1.9-88.8) \mathrm{ng} / \mathrm{mL}$, respectively, $p=$ $0.002)$. Although total cholesterol levels were weakly correlated with UACR $(r=0.172, p=0.059)$, there were no correlations between UACR and the other parameters including systolic and diastolic blood pressures. There was still significant association between YKL-40 levels and UACR after adjustment for diabetes duration which related both of them $(r=0.185, p=0.029)$. To demonstrate whether serum YKL-40 levels are a determinant of UACR, we performed a stepwise multivariate regression analysis including UACR as a dependent variable and following variables as explanatory variables: gender (male $=1$, female $=0)$, diabetes duration, $\mathrm{HbAlc}$, total cholesterol, creatinine and YKL-40 levels, all of which were associated with UACR in the above mentioned univariate analyses. This analysis showed that YKL-40 levels were independent risk factors for UACR as well as gender and HbAlc levels (Table 2). Furthermore, in a multivariate regression analysis including conventional risk factors: age, smoking status, blood pressure, $\mathrm{HbA1c}$, total cholesterol, creatinine levels [12], YKL-40 levels were still independent determinant for UACR $(\beta$ $=0.357, p=0.042$ ) as well as HbA1c.

In addition, YKL-40 levels were significantly higher in participants with diabetic retinopathy as compared to those without it (median (range) 55.5 (23.3-274.1) and 50.3(21.4-237.4) ng/mL, respectively, $p=0.039$ ). To evaluate that YKL-40 levels are associated with diabetic retinopathy even after adjustment of established risk factors (i.e. diabetes duration, blood pressure, $\mathrm{HbA} 1 \mathrm{c})$, we performed a logistic regression analysis. Although this analysis revealed that diabetes duration $(\mathrm{OR}=1.19(95 \% \mathrm{CI}: 1.09-1.30), p<0.001)$ and HbAlc $(\mathrm{OR}=1.47$ (95\% CI: 1.07-2.00), $p=0.016)$ were significant and independent risk factors for retinopathy, YKL-40 levels were not a significant determinant after adjustment of these parameters $(\mathrm{OR}=1.35$ (95\% CI 0.59-3.10), $p=0.475$ ).

There were no associations between estimated GFR, CVR-R, carotid intima-media thickness evaluated by ultrasonography and YKL-40 levels (data not shown).

\section{Relation between serum YKL-40 and hs-CRP levels}

We also evaluated the relation between YKL-40 and hs-CRP levels which is one of the established inflammatory markers in our participants. Hs-CRP levels in type 1 diabetic patients were significantly higher than control groups (median (range) 321 (51-13000) and 171 (53-7380) ng/mL, respectively, $p=0.001$ ) (Table 
1). There were significant association between YKL-40 and hs-CRP levels in type 1 diabetic patients $(r=0.188$, $p=0.040)$ as well as control groups. Although hsCRP levels were significantly associated with BMI $(r=$ $0.203, p=0.026)$ and DBP $(r=0.244, p=0.007)$, there was no association with UACR as well as other diabetic complications in our participants (data not shown).

\section{Discussion}

Although the incidence and prevalence of type 1 diabetes are much lower in Japanese than in Caucasoid populations [13], unstable glycemic control and the progression of diabetic complications are major problems in both populations. In addition, since there are a few opportunity that type 1 diabetic patients can receive pancreas transplantation to cure diabetes mellitus in Japan compared to Western countries [14], it is very important to predict the development of microand macrovascular complications in early stage.

In the present study, we found that YKL-40 levels were significantly elevated in young Japanese type 1 diabetic patients without severe diabetic complications as compared to age- and gender-matched healthy controls, and that elevated YKL-40 levels were significantly associated with increased albuminuria in these patients.

These findings are consistent with several previous studies. Rathcke et al. reported that YKL-40 levels were elevated in type 1 diabetic patients and increased with levels of albuminuria [8]. Their findings were similar to ours, but it is noteworthy that our participants were Asian, younger, shorter diabetes duration and less diabetic complications than their participants: Caucasian, middle-aged (about 50 years old), longer diabetes duration (over 30 years) and more advanced diabetic complications. Similarly, Røndbjerg et al. showed that type 2 diabetic patients with macroalbuminuria (UACR > $25 \mathrm{mg} / \mathrm{mmol}$ ) had higher YKL-40 levels [15], whereas most of our participants had normoalbuminuria (UACR $<30 \mathrm{mg} / \mathrm{g} \cdot \mathrm{Cr}$ ) and only twelve patients $(9.1 \%)$ had level of over microalbuminuria (UACR $\geq 30 \mathrm{mg} / \mathrm{g} \cdot \mathrm{Cr}$ ). These differences raise the possibility that YKL-40 can predict diabetic nephropathy in earlier stage. Furthermore, this is the first report to show that YKL-40 levels were significantly higher in type 1 diabetic patients with retinopathy as compared to those without it.

The complete biological function of YKL-40 is unclear, but many studies have suggested that YKL-40 has a role in inflammation and remodeling of the extra- cellular matrix [1]. Clinical studies showed that biomarkers of inflammation and endothelial dysfunction were higher in type 1 diabetic patients with diabetic complications $[16,17]$ and that chronic low-grade inflammation and endothelial dysfunction were associated with the occurrence and progression of diabetic microangiopathy including nephropathy [16-18] and retinopathy [19]. Thus, it is possible that YKL-40 plays an important role in the pathogenesis of diabetic microangiopathy via intermediating low-grade inflammation and endothelial dysfunction.

The presence of albuminuria is not only a predictor of progression of nephropathy and decline of estimated GFR in type 1 diabetic patients [20,21], but also a risk factor for cardiovascular disease with or without diabetes mellitus [22]. Inflammation and endothelial dysfunction are thought to be key processes in the progression of atherosclerosis. In vitro studies have demonstrated that YKL-40 modulates vascular endothelial cell morphology by promoting the formation of branching tubule, indicating that YKL-40 may function in angiogenesis by stimulating the migration and reorganization of vascular endothelial cells. Furthermore, YKL-40 promotes vascular smooth muscle cell attachment, spreading and migration, suggesting that YKL-40 has a role in the process of atherosclerotic plaque formation where smooth muscle cells are induced to migrate through the intima in response to exogenous signals [1]. Clinical reports showed that YKL-40 had association with morbidity and mortality of cardiovascular diseases [4, 23]. Alternatively, YKL-40 levels were particularly higher in patients with symptomatic carotid plaques accompanied with increasing mRNA YKL-40 levels within the atherosclerosis lesion, suggesting that YKL-40 might be a marker of plaque instability [24]. Both elevated YKL-40 levels and albuminuria may reflect a pathophysiological process predisposing cardiovascular disease. However, since our type 1 diabetic patients did not have cardiovascular disease and we could not find any significant correlation between YKL-40 levels and carotid intima-media thickness which is a surrogate marker of atherosclerosis in this cross-sectional study, longitudinal study would be necessary to evaluate possible association between YKL-40 levels and the progression of atherosclerosis.

We also examined the relationship between YKL-40 and hs-CRP levels in this study. Although a significantly positive correlation between YKL-40 and hsCRP levels were observed reflecting the underlying inflammation and endothelial dysfunction, there was 
no association between hs-CRP levels and albuminuria. Several studies have shown that hs-CRP levels were significantly higher in type 1 diabetic patients with microalbuminuria than those with normoalbuminuria $[25,26]$. This result may reflect the phenomenon that serum YKL-40 levels increase more sensitively to focal inflammation and endothelial dysfunction as compared to serum hs-CRP levels. These might be caused by difference in production process between hs-CRP and YKL-40; hs-CRP is produced and secreted from the liver in response to the proinflammation cytokines such as IL- 6 and TNF- $\alpha$, while YKL-40 is produced at the focal inflammation or atherosclerosis sites stimulated by IL-6, IL-17 and IL-18 [1, 27].

Thus, our present findings are in accordance with these previous basic and clinical findings and suggest that YKL-40 can be a new biomarker to identify type 1 diabetic patients with ongoing low-grade inflammation and endothelial dysfunction who would likely develop diabetic microangiopathy.

There are several limitations in this study. First, this is a cross-sectional study with a relatively small number of subjects. Second, because of the cross-sectional nature of this study, the associations do not necessarily indicate causality. Third, it should be evaluated in fur- ther studies whether serum YKL-40 is more sensitive marker of focal inflammation in the kidneys as compared to serum hs-CRP. Pathophysiological research including renal biopsy accompanied by measurement of serum inflammatory markers would strengthen this hypothesis. Fourth, measurement of urinary YKL-40 levels may also shed light on the clarification of the possible role of YKL-40 in the development of albuminuria, although we failed to measure them due to lack of urine samples. Finally, although we tried to minimize possible influence of known major confounders, the results may have been confounded by unknown factors. Therefore, we need prospective cohort studies with a larger sample size to prove whether YKL-40 levels can be a predictor of diabetic complications.

In summary, our results indicate that serum YKL-40 levels have a linear association with albuminuira and are elevated in participants with retinopathy in young Japanese type 1 diabetic patients. These data suggest that YKL-40 levels could be a tool to assess the risk of diabetic microangiopathy in the very early stage in type 1 diabetic patients. Further longitudinal studies are needed to confirm that YKL-40 can predict the development of diabetic complications.

\section{References}

1. Johansen JS (2006) Studies on serum YKL-40 as a biomarker in diseases with inflammation, tissue remodeling, fibrosis and cancer. Dan Med Bull 53: 172-209.

2. Johansen JS, Stoltenberg M, Hansen M, Florescu A, Hørslev-Petersen K (1999) Serum YKL-40 concentrations in patients with rheumatoid arthritis: relation to disease activity. Rheumatology (Oxford) 38: 618-626.

3. Johansen JS, Bojesen SE, Mylin AK, Frikke-Schmidt R, Price PA (2009) Elevated plasma YKL-40 predicts increased risk of gastrointestinal cancer and decreased survival after any cancer diagnosis in the general population. J Clin Oncol 27: 572-578.

4. Kastrup J, Johansen JS, Winkel P, Hansen JF, Hildebrandt P; CLARICOR Trial Group (2009) High serum YKL-40 concentration is associated with cardiovascular and allcause mortality in patients with stable coronary artery disease. Eur Heart J 30: 1066-1072.

5. Rathcke CN, Johansen JS, Vestergaard H (2006) YKL-40, a biomarker of inflammation, is elevated in patients with type 2 diabetes and is related to insulin resistance. Inflamm Res 55: 53-59.

6. Nielsen AR, Erikstrup C, Johansen JS, Fischer
CP, Plomgaard P (2008) Plasma YKL-40: a BMIindependent marker of type 2 diabetes. Diabetes 57: 3078-3082.

7. Yasuda T, Kaneto H, Katakami N, Kuroda A, Matsuoka TA (2011) YKL-40, a new biomarker of endothelial dysfunction, is independently associated with albuminuria in type 2 diabetic patients. Diabetes Res Clin Pract 91: e50-e52.

8. Rathcke CN, Persson F, Tarnow L, Rossing P, Vestergaard H (2009) YKL-40, a marker of inflammation and endothelial dysfunction, is elevated in patients with type 1 diabetes and increases with levels of albuminuria. Diabetes Care 32: 323-328.

9. Kawamori R (1992) Prevalence of carotid atherosclerosis in diabetic patients: ultrasound high-resolution B-mode imaging on carotid arteries. Diabetes Care 15: 1290-1294.

10. The Committee of the Japan Diabetes Society on the Diagnostic Criteria of Diabetes Mellitus (2010) Report of the Committee on the Classification and Diagnostic Criteria of Diabetes Mellitus. J Diabetes Invest 1: 212228. 
11. Matsuo S, Imai E, Horio M, Yasuda Y, Tomita K; Collaborators developing the Japanese equation for estimated GFR (2009) Revised equations for estimated GFR from serum creatinine in Japan. Am J Kidney Dis 53: 982-992.

12. Reutens AT, Atkins RC (2011) Epidemiology of diabetic nephropathy. Contrib Nephrol 170: 1-7.

13. Kawasaki E, Matsuura N, Eguchi K (2006) Type 1 diabetes in Japan. Diabetologia 49: 828-836.

14. Egawa H, Tanabe K, Fukushima N, Date H, Sugitani A (2012) Current status of organ transplantation in Japan. Am J Transplant 12: 523-530.

15. Røndbjerg AK, Omerovic E, Vestergaard H (2011) YKL-40 levels are independently associated with albuminuria in type 2 diabetes. Cardiovasc Diabetol 10: 54.

16. Schram MT, Chaturvedi N, Schalkwijk CG, Fuller JH, Stehouwer CD (2005) Markers of inflammation are cross-sectionally associated with microvascular complications and cardiovascular disease in type 1 diabetes: the EURODIAB Prospective Complications Study. Diabetologia 48: 370-378.

17. Astrup AS, Tarnow L, Pietraszek L, Schalkwijk CG, Stehouwer CD (2008) Markers of endothelial dysfunction and inflammation in type 1 diabetic patients with or without diabetic nephropathy followed for 10 years: association with mortality and decline of glomerular filtration rate. Diabetes Care 31: 1170-1176.

18. Lin J, Glynn RJ, Rifai N, Manson JE, Ridker PM (2008) Inflammation and progressive nephropathy in type 1 diabetes in the diabetes control and complications trial. Diabetes Care 31: 2338-2343.

19. Klein BE, Knudtson MD, Tsai MY, Klein R (2009) The relation of markers of inflammation and endothelial dysfunction to the prevalence and progression of diabetic retinopathy: Wisconsin epidemiologic study of diabetic retinopathy. Arch Ophthalmol 127: 1175-1182.

20. Rossing P, Hougaard P, Parving HH (2002) Risk factors for development of incipient and overt diabetic nephropathy in type 1 diabetic patients: a 10-year prospective observational study. Diabetes Care 25: 859-864.

21. Molitch ME, Steffes M, Sun W, Rutledge B, Cleary $\mathrm{P}$; Epidemiology of Diabetes Interventions and Complications Study Group (2010) Development and progression of renal insufficiency with and without albuminuria in adults with type 1 diabetes in the diabetes control and complications trial and the epidemiology of diabetes interventions and complications study. Diabetes Care 33: 1536-1543.

22. Gerstein HC, Mann JF, Yi Q, Zinman B, Dinneen SF, Hoogwerf B; HOPE Study Investigators (2001) Albuminuria and risk of cardiovascular events, death, and heart failure in diabetic and nondiabetic individuals. JAMA 286: 421-426.

23. Kastrup J (2012) Can YKL-40 be a new inflammatory biomarker in cardiovascular disease? Immunobiology 217: 483-491.

24. Michelsen AE, Rathcke CN, Skjelland M, Holm S, Ranheim T (2010) Increased YKL-40 expression in patients with carotid atherosclerosis. Atherosclerosis 211:589-595.

25. Marcovecchio ML, Giannini C, Widmer B, Dalton RN, Martinotti S (2008) C-reactive protein in relation to the development of microalbuminuria in type 1 diabetes: the Oxford Regional Prospective Study. Diabetes Care 31: 974-976.

26. Devaraj S, Cheung AT, Jialal I, Griffen SC, Nguyen D (2007) Evidence of increased inflammation and microcirculatory abnormalities in patients with type 1 diabetes and their role in microvascular complications. Diabetes 56: 2790-2796.

27. Nielsen AR, Plomgaard P, Krabbe KS, Johansen JS, Pedersen BK (2011) IL-6, but not TNF- $\alpha$, increases plasma YKL-40 in human subjects. Cytokine 55: 152155. 